\title{
Scaled Dimension and the Kolmogorov Complexity of Turing-Hard Sets
}

\author{
John M. Hitchcock* $\quad$ María López-Valdés ${ }^{\dagger} \quad$ Elvira Mayordomo ${ }^{\ddagger}$
}

\begin{abstract}
We study constructive and resource-bounded scaled dimension as an information content measure and obtain several results that parallel previous work on unscaled dimension. Scaled dimension for finite strings is developed and shown to be closely related to Kolmogorov complexity. The scaled dimension of an infinite sequence is characterized by the scaled dimensions of its prefixes. We obtain an exact Kolmogorov complexity characterization of scaled dimension.

Juedes and Lutz (1996) established a small span theorem for $\mathrm{P} /$ poly-Turing reductions which asserts that for any problem $A$ in ESPACE, either the class of problems reducible to $A$ (the lower span) or the class of problems to which $A$ is reducible (the upper span) has measure 0 in ESPACE. We apply our Kolmogorov complexity characterization to improve this to $(-3)^{\mathrm{rd}}$ order scaled dimension 0 in ESPACE. As a consequence we obtain a new upper bound on the Kolmogorov complexity of Turing-hard sets for ESPACE.
\end{abstract}

\section{Introduction}

Fractal dimension is closely related to measures of information content. For example, Ryabko [28, 29], Staiger [30, 31], and Cai and Hartmanis [5] proved results relating Hausdorff dimension to Kolmogorov complexity. This relation becomes an equivalence with Lutz's effective fractal dimensions [25, 26]: constructive dimension has a characterization in terms of Kolmogorov complexity $[26,27]$ and computable and space-bounded dimension in terms of space-bounded Kolmogorov complexity [8]. While Kolmogorov complexity is a measure over finite strings, dimension is defined for sets of infinite sequences. Thus, these relations between dimension and Kolmogorov complexity are relations between the dimension of an infinite sequences and the Kolmogorov complexity of its prefixes.

Lutz [26] also developed dimension of finite strings as a discrete version of constructive dimension. He proved that $(i)$ the Kolmogorov complexity of a string is the product of its length and its dimension, and (ii) the constructive dimension of an infinite sequence is characterized by the dimensions of its prefixes. Taken together, $(i)$ and $(i i)$ constitute a proof of the Kolmogorov complexity characterization of constructive dimension.

Scaled dimension [12] is an extension of effective dimension defined by introducing scales at which dimension may be measured. It was developed to quantify the difference in the size of some

\footnotetext{
*Department of Computer Science, University of Wyoming, USA. jhitchco@cs.uwyo.edu. This research was supported in part by National Science Foundation grant 0515313. Part of this research was done while this author was visiting the University of Zaragoza.

${ }^{\dagger}$ Departamento de Informática e Ingeniería de Sistemas, María de Luna 1, Universidad de Zaragoza, 50018 Zaragoza, SPAIN. marlopez@unizar.es, elvira at unizar dot es. This research was supported in part by Spanish Government MEC projects TIC 2002-04019-C03-03 and TIN 2005-08832-C03-02.
} 
complexity classes. For example, classes such as $\operatorname{SIZE}\left(2^{\alpha n}\right)$ or SIZE $\left(2^{n^{\alpha}}\right)$ are not distinguished by unscaled dimension because they all have dimension 0 . Scaled dimension precisely quantifies the difference among these circuit-size classes [12] and has several other applications in complexity theory $[7,10,11,13,14,15]$.

In this paper, we develop relationships between scaled dimension and Kolmogorov complexity that parallel the previous results on unscaled dimension.

- We develop scaled dimension for finite strings by rescaling Lutz's dimension of finite strings. For every integer $k$, each sufficiently large string $w$ has a scaled dimension $\operatorname{dim}^{(k)}(w)$. We show that the scaled dimension of a string is closely related to its Kolmogorov complexity:

$$
\left|K(w)-g_{k}\left(|w|, \operatorname{dim}^{(k)}(w)\right)\right|=O(1) .
$$

Here for each $k, g_{k}$ is a sublinear function defined in section 2. We also prove that the scaled dimension of an infinite sequence $S$ can be obtained from the scaled dimensions of its prefixes:

$$
\operatorname{dim}^{(k)}(S)=\liminf _{n \rightarrow \infty} \operatorname{dim}^{(k)}(S[0 . . n-1]) .
$$

- We prove that the constructive, computable, and space-bounded scaled dimensions can be interpreted as information content measures and we show how the scale influences this interpretation. For example, the $(-1)^{\mathrm{st}}$-scaled pspace-dimension of a class $X$ is the smallest $s$ for which there is a $c$ such that for every $A \in X$,

$$
K S^{m^{c}}(A[0 . . m-1]) \leq m-m^{1-s} \quad \text { i.o. } m .
$$

Equivalently, the $(-1)^{\text {st }}$-order scaled pspace-dimension of $X$ is the smallest $s$ for which there is a $c$ that for every $A \in X$,

$$
K S^{2^{c n}}\left(A_{\leq n}\right) \leq 2^{n+1}-2^{n(1-s)} \quad \text { i.o. } n .
$$

This means that the $(-1)^{\text {st }}$-scaled pspace-dimension of $X$ is directly related to the smallest i.o. upper bound (equivalently, to the largest a.e. lower bound) of the form $2^{n+1}-2^{n \alpha}$ on the space-bounded Kolmogorov complexity of all languages in $X$. By this characterization, each scaled dimension result can be interpreted as a Kolmogorov complexity upper bound.

Juedes and Lutz [19] proved a small span theorem for $\mathrm{P} /$ poly-Turing reductions in ESPACE. This theorem says that for any $A \in \mathrm{ESPACE}$, either the class of languages reducible to $A$ (the lower span) or the class of problems to which $A$ can be reduced (the upper span) has measure 0 in ESPACE. We improve this theorem by replacing measure with $(-3)^{\mathrm{rd}}-$ scaled dimension. The proof uses our Kolmogorov complexity characterization of scaled dimension. This result also subsumes the scaled dimension small span theorem for polynomial-time many-one reductions in ESPACE [10].

Our small span theorem implies that the class of $\leq_{\mathrm{T}}^{\mathrm{P} / \text { poly }}$-hard sets for ESPACE has $(-3)^{\mathrm{rd}}$ scaled pspace-dimension 0. From this it follows that every $\leq_{\mathrm{T}}^{\mathrm{P} / \text { poly }}$-hard set has unusually low space-bounded Kolmogorov complexity. The upper bound we give matches the bound of Juedes of Lutz [19] for the $\leq_{\mathrm{m}}^{\mathrm{P} / \text { poly }}$-hard sets.

The paper is organized as follows. Section 2 outlines notation and preliminaries. Section 3 develops scaled dimension of finite strings and studies its relation with Kolmogorov complexity and constructive scaled dimension. Section 4 describes our characterization and section 5 presents our results for the Kolmogorov complexity of hard sets. 


\section{Preliminaries}

A string is a finite and binary sequence $w \in\{0,1\}^{*}$. Let $|w|$ denote the length of a string and $\lambda$ denote the empty string. The Cantor space $\mathbf{C}$ is the set of all infinite binary sequences. Let $x[i . . j]$ for $0 \leq i \leq j$ denote the $i$-th through the $j$-th bits of $x$, where $x \in\{0,1\}^{*} \cup \mathbf{C}$. Let $w x$ denote the concatenation of the string $w$ and the string or sequence $x$. Let $w \sqsubseteq x$ denote that $w$ is a prefix of $x$. Let $\mathcal{T}$ be the set of all terminated binary strings and prefixes, that is

$$
\mathcal{T}=\{0,1\}^{*} \cup\{0,1\}^{*} \square,
$$

where the symbol $\square$ is used to mark the end of a string.

Let $s_{0}, s_{1}, s_{2} \ldots$ be the standard enumeration of $\{0,1\}^{*}$ in lexicographical order. We identify each language with its characteristic sequence $\chi_{A} \in \mathbf{C}$ where

$$
\chi_{A}[i]= \begin{cases}1 & \text { if } s_{i} \in A \\ 0 & \text { if } s_{i} \notin A\end{cases}
$$

For each $n \in \mathbb{N}$, let

$$
\begin{array}{ccl}
A_{=n}=A \cap\{0,1\}^{n} & \text { or } & A_{=n}=A\left[2^{n}-1 . .2^{n+1}-2\right], \\
A_{\leq n}=A \cap\{0,1\}^{\leq n} & \text { or } & A_{\leq n}=A\left[0 . .2^{n+1}-2\right],
\end{array}
$$

depending on the context. For each $i \in \mathbb{N}$, let $G_{i}$ be the class of functions from $\mathbb{N}$ to $\mathbb{N}$ defined by

$$
\begin{aligned}
G_{0} & =\left\{f \mid(\exists k)\left(\forall^{\infty} n\right) f(n) \leq k n\right\} . \\
G_{i+1} & =\left\{f \mid\left(\exists g \in G_{i}\right)\left(\forall^{\infty} n\right) f(n) \leq 2^{g(\log n)}\right\} .
\end{aligned}
$$

Let $\Delta$ denote any of the following classes of total functions,

$$
\begin{aligned}
\text { all } & =\left\{f \mid f:\{0,1\}^{*} \rightarrow\{0,1\}^{*}\right\} \\
\text { comp } & =\{f \in \text { all } \mid f \text { is computable }\} \\
\mathrm{p}_{i} \text { space } & =\left\{f \in \text { all } \mid f \text { is computable in } G_{i} \text { space }\right\}(i \geq 1)
\end{aligned}
$$

and let $R(\Delta)$ denote any of the following complexity classes,

$$
\begin{aligned}
R(\text { all }) & =\mathbf{C} \\
R(\text { comp }) & =\mathrm{DEC} \\
R\left(\mathrm{p}_{i} \text { space }\right) & =\mathrm{E}_{i} \operatorname{SPACE}=\operatorname{DSPACE}\left(2^{G_{i-1}}\right) \text { for } i \geq 1 .
\end{aligned}
$$

Let pspace and ESPACE denote $\mathrm{p}_{1}$ space and $\mathrm{E}_{1} \mathrm{SPACE}$ respectively.

Definition. Let $D$ be a discrete domain such as $\mathbb{N},\{0,1\}^{*}$ or $\mathcal{T}$ and let $f: D \rightarrow[0, \infty)$.

1. $f$ is $\Delta$-computable if there exists a function $\widehat{f}: D \times \mathbb{N} \rightarrow \mathbb{Q} \cap[0, \infty)$ in $\Delta$ such that for all $(w, n) \in D \times \mathbb{N},|\widehat{f}(w, n)-f(w)| \leq 2^{-n} \quad$ (with $n$ coded in unary and the output coded in binary).

2. $f$ is exactly $\Delta$-computable if $f: D \rightarrow \mathbb{Q} \cap[0, \infty)$ and $f \in \Delta$.

3. $f$ is constructive (or lower semicomputable) if there is a computable function $\widehat{f}: D \times \mathbb{N} \rightarrow$ $\mathbb{Q} \cap[0, \infty)$ such that, 
(a) $\widehat{f}(w, n) \leq \widehat{f}(w, n+1)<f(w)$, for all $(w, n) \in D \times \mathbb{N}$,

(b) $\lim _{n \rightarrow \infty} \widehat{f}(w, n)=f(w)$, for all $w \in D$.

\section{Definition.}

1. A series $\sum_{n=0}^{\infty} a_{n}$ of nonnegative real numbers $a_{n}$ is $\Delta$-convergent if there is a function $h$ : $\mathbb{N} \rightarrow \mathbb{N}$ such that $h \in \Delta$ and

$$
\sum_{n=h(r)}^{\infty} a_{n} \leq 2^{-r}
$$

for all $r \in \mathbb{N}$. Such a function $h$ is called a modulus of the convergence.

2. A sequence

$$
\sum_{n=0}^{\infty} a_{j, n}(j=0,1,2, \ldots)
$$

of series of nonnegative real numbers is uniformly $\Delta$-convergent if there is a function $f: \mathbb{N}^{2} \rightarrow$ $\mathbb{N}$ such that $f \in \Delta$ and $f_{j}$ is a modulus of the convergence of the series $\sum_{n=0}^{\infty} a_{j, n}$ for every $j \in \mathbb{N}$, where $f_{j}(n)=f(j, n)$ for every $j$ and $n$.

\subsection{Scaled Dimension}

Scaled dimension is defined using functions called scaled gales. These functions are a rescaled version of the more familiar concept of gales [25]. The main concept in the definition of scaled gales is a scale, which is a function $g:(a, \infty) \times[0, \infty) \rightarrow \mathbb{R}$. A scale must satisfy certain properties that are given in [12] and will not be discussed here. The most important family of scale functions $g_{i}:\left(a_{i}, \infty\right) \times[0, \infty) \rightarrow \mathbb{R}$ and $g_{-i}:\left(a_{i}, \infty\right) \times[0, \infty) \rightarrow \mathbb{R}$ is defined by the following recursion on $i \in \mathbb{N}$.

- $g_{0}(m, s)=m s$.

- $g_{i+1}(m, s)=2^{g_{i}(\log m, s)}$.

- $g_{-i}(m, s)= \begin{cases}m+g_{i}(m, 0)-g_{i}(m, 1-s) & \text { for } 0 \leq s \leq 1 \\ g_{i}(m, s) & \text { for } s>1\end{cases}$

The domain of $g_{i}$ coincides with that of $g_{-i}$ and $a_{i}$ is given for the recursion $a_{0}=-\infty$ and $a_{i+1}=2^{a_{i}}$. Let $m_{i}$ denote $\max \left\{a_{i}, 0\right\}$.

Definition. Let $s \in[0, \infty)$, let $g:(a, \infty) \times[0, \infty) \rightarrow \mathbb{R}$ be a scale function and let $m=\max \{a, 0\}$.

1. An s-supergale is a function $d:\{0,1\}^{*} \rightarrow[0, \infty)$ such that for all $w \in\{0,1\}^{*}$,

$$
d(w) \geq 2^{-s}[d(w 0)+d(w 1)] .
$$

2. A $g$-scaled $s$-supergale (briefly, an $s^{g}$-gale) is a function $d:\{0,1\}^{*} \rightarrow[0, \infty)$ such that for all $w \in\{0,1\}^{>m}$,

$$
d(w) \geq 2^{-\Delta g(|w|, s)}[d(w 0)+d(w 1)],
$$

where $\Delta g:(a, \infty) \times(a, \infty) \rightarrow \mathbb{R}$ is defined by

$$
\Delta g(m, s)=g(m+1, s)-g(m, s) .
$$


3. An $s$-gale (a g-scaled $s$-gale) is a function $d:\{0,1\}^{*} \rightarrow[0, \infty)$ that verifies equation $2.1(2.2)$ with equality.

An $s$-gale can be interpreted as a strategy for betting on the successive bits of a binary string. The fairness of the gambling game depends on $s$. An $s^{g}$-gale can also be interpreted as a betting strategy, but in this case, the fairness of the gambling depends on the scale $g$ and on $s$. The notion of success corresponds to getting unbounded capital in this game.

Definition. Let $d:\{0,1\}^{*} \rightarrow[0, \infty)$ be an $s^{g}$-supergale,

1. $d$ succeeds on a language $A \subseteq\{0,1\}^{*}$ if

$$
\limsup _{n \rightarrow \infty} d(A[0 . . n-1])=\infty .
$$

2. The success set of $d$ is

$$
S^{\infty}[d]=\left\{A \subseteq\{0,1\}^{*} \mid d \text { succeeds on } A\right\} .
$$

3. The unitary success set of $d$ is

$$
S^{1}[d]=\{A \in \mathbf{C} \mid \exists n d(A[0 . . n-1]) \geq 1\} .
$$

We are ready to define scaled dimension now.

Definition. Let $X \subseteq \mathbf{C}$ and let $g:(a, \infty) \times[0, \infty) \rightarrow \mathbb{R}$ be a scale function.

1. The $g$-scaled $\Delta$-dimension of $X$ is

$$
\operatorname{dim}_{\Delta}^{g}(X)=\inf \left\{s \mid \text { there exists a } \Delta \text {-computable } s^{g} \text {-supergale } d \text { s.t. } X \subseteq S^{\infty}[d]\right\} .
$$

2. The $g$-scaled $\Delta$-dimension of $X$ within $R(\Delta)$ is

$$
\operatorname{dim}_{\Delta}^{g}(X \mid R(\Delta))=\operatorname{dim}_{\Delta}^{g}(X \cap R(\Delta)) .
$$

3. The $g$-scaled constructive dimension of $X$ is

$$
\operatorname{cdim}^{g}(X)=\inf \left\{s \mid \text { there exists a constructive } s^{g} \text {-supergale } d \text { s.t. } X \subseteq S^{\infty}[d]\right\} .
$$

Lutz [25] and Hitchcock [9] proved respectively that resource-bounded and constructive dimension can be defined in terms of gales.

Notice that the definition of scaled gales coincides with the definition of gales when the scale $g_{0}$ is considered. For this scale functions, $g_{0}(m+1, s)-g_{0}(m, s)=s$ and there is not dependence on $m$. In particular $\operatorname{dim}_{\text {all }}^{g_{0}}(X)=\operatorname{dim}_{\mathrm{H}}(X)$ is the Hausdorff dimension of $X ; \operatorname{cdim}_{\text {all }}^{g_{0}}(X)=\operatorname{cdim}(X)$ is the constructive dimension of $X$ as defined in [26]; and $\operatorname{dim}_{\Delta}^{g_{0}}(X)=\operatorname{dim}_{\Delta}(X)$ is the resource-bounded dimension as defined in [25].

In this paper we restrict the gales to the family above but our results can be extended to other families of scale functions.

Notation. Let $k \in \mathbb{Z}$,

1. $s^{(k)}$-gale denotes the $s^{g_{k}}$-gale. 
2. $\operatorname{dim}_{\Delta}^{(k)}$ denotes the $k^{\text {th }}$-order scaled $\Delta$-dimension $\operatorname{dim}_{\Delta}^{g_{k}}$.

3. $\operatorname{cdim}^{(k)}$ denotes the $k^{\text {th }}$-order scaled constructive dimension $\operatorname{cdim}^{g_{k}}$.

The next family of functions will be used in several results.

Definition. Let $i \in \mathbb{N}$. Let $f_{i}: \mathbb{N} \times[0, \infty) \rightarrow \mathbb{R}$ and $f_{-i}: \mathbb{N} \times[0, \infty) \rightarrow \mathbb{R}$ be defined by

$$
\begin{aligned}
f_{i}(n, x) & =\frac{\log ^{(i)} x}{\log ^{(i)} n} \\
\left(f_{-i}\right)(n, x) & =1-\frac{\log ^{(i)}\left(n-x-g_{i}(n, 0)\right)}{\log ^{(i)} n}
\end{aligned}
$$

where $\log ^{(i)}$ denotes the $i$-time iterated applications of log, that is,

$$
\log ^{(i)}(x)=\overbrace{\log (\ldots \log x}^{i} x)
$$

Notice that for each $k \in \mathbb{Z}, x \in[0, \infty), f_{k}(\cdot, x)$ is the inverse of $g_{k}(\cdot, x)$.

\subsection{Kolmogorov Complexity}

We next review the Kolmogorov complexity, some of its variants and some well-known results (for more details see the textbook by Li and Vitányi [22]).

Definition. Fix a universal Turing machine $U$. Let $t: \mathbb{N} \rightarrow \mathbb{N}$ and $w \in\{0,1\}^{*}$.

1. The Kolmogorov complexity of $w$ is

$$
K(w)=\min \{|\pi| \mid U(\pi)=w\} .
$$

2. The $t$-space bounded Kolmogorov complexity of $w$ is

$$
K S^{t}(w)=\min \{|\pi| \mid U(\pi)=w \text { in } \leq t(|w|) \text { space }\} .
$$

The above definition is (additively) invariant of the choice of the universal machine $U$ [22].

\section{Definition.}

1. A subprobability measure on $\{0,1\}^{*}$ is a function $p:\{0,1\}^{*} \rightarrow[0,1]$ such that

$$
\sum_{w \in\{0,1\}^{*}} p(w) \leq 1
$$

2. A constructive subprobability measure $p$ on $\{0,1\}^{*}$ is optimal if for every constructive subprobability measure $p^{\prime}$ there is a real constant $\alpha>0$ such that, for all $w \in\{0,1\}^{*}, p(w)>$ $\alpha p^{\prime}(w)$. 
Theorem 2.1. (Levin [32]) There exists an optimal constructive subprobability measure $\mathbf{m}$ on $\{0,1\}^{*}$.

The following theorem is the well-known characterization by Levin $[20,21]$ and Chaitin [6] of Kolmogorov complexity in terms of $\mathbf{m}$. Further details may be found in [22].

Theorem 2.2. There is a constant $c \in \mathbb{N}$ such that for all $w \in\{0,1\}^{*}$,

$$
\left|K(w)-\log \frac{1}{\mathbf{m}(w)}\right| \leq c
$$

\section{Scaled Dimension of Finite Strings}

In this section we extend the dimension of finite strings [26] to scaled dimension of finite strings. We achieve this by introducing scaled termgales, variants of scaled gales adapted to terminating strings, and then showing the existence of an optimal constructive scaled termgale. This allows us to give a universal definition of the scaled dimension of a string. Finally, we characterized the scaled dimension of a sequence in terms of the scaled dimension of its finite prefixes. In the next section we will use this result to give a Kolmogorov characterization of constructive scaled dimension and we also give a Kolmogorov characterization of resource-bounded scaled dimension through an alternative proof. Our proofs differ from [26] due to the dependence on the scale function.

Our notion of termgale is an extension of gales to terminating strings.

Definition. Let $s \in[0, \infty)$ and $k \in \mathbb{Z}$,

1. An $s$-termgale is a function $d: \mathcal{T} \rightarrow[0, \infty)$ such that

(a) $d(\lambda) \leq 1$

(b) For all $w \in\{0,1\}^{*}$,

$$
d(w) \geq 2^{-s}[d(w 0)+d(w 1)+d(w \square)] .
$$

2. An $s^{(k)}$-termgale is a function $d: \mathcal{T} \rightarrow[0, \infty)$ such that

(a) For all $w \in\{0,1\}^{\leq m_{|k|}}, d(w) \leq 1$.

(b) For all $w \in\{0,1\}^{>m_{|k|}}$,

$$
d(w) \geq 2^{-\triangle g_{k}(|w|, s)}[d(w 0)+d(w 1)+d(w \square)] .
$$

Remember that $m_{k}:=\max \left\{a_{k}, 0\right\}$, where $\left(a_{k}, \infty\right)$ is the domain of $g_{k}$.

Remark. As in the case of $s^{(k)}$-gales, an $s^{(k)}$-termgale is a strategy for betting on the successive bits of a binary string but also on the point where the string terminates. The fairness of the gambling game depends on the scale function $g_{k}$. In the case of an $s$-termgale, the fairness of the game depends only on $s$. An $s^{0}$-termgales is an $s$-termgale.

Observation 3.1. Let $k \in Z$, let $s, t \in[0, \infty)$ and let $d, d^{\prime}: \mathcal{T} \rightarrow[0, \infty)$. If

$$
2^{-g_{k}(|w|, s)} d(w)=2^{-g_{k}(|w|, t)} d^{\prime}(w),
$$

for all $w \in \mathcal{T}$ with $|w|>m_{|k|}$, then $d$ is an $s^{(k)}$-termgale if and only if $d^{\prime}$ is a $t^{(k)}$-termgale. 
Due to this observation, each $0^{(k)}$-termgale determines a whole family of $s^{(k)}$-termgales that will be used in the definition of scaled dimension.

Definition. 1. A $k$-termgale is a family $d=\left\{d^{s} \mid s \in[0, \infty)\right\}$ such that each $d^{s}$ is an $s^{(k)}$ termgale and for all $s, t \in[0, \infty), w \in \mathcal{T}$ with $|w|>m_{|k|}$,

$$
2^{-g_{k}(|w|, s)} d^{s}(w)=2^{-g_{k}(|w|, t)} d^{t}(w) .
$$

2. A $k$-termgale $d$ is constructive if $d^{0}$ is constructive.

3. A constructive $k$-termgale $\tilde{d}$ is optimal if for every constructive $k$-termgale $d$ there is a constant $\alpha>0$ such that for all $s \in[0, \infty)$ and $w \in\{0,1\}^{>m_{|k|}}$,

$$
\tilde{d}^{s}(w \square)>\alpha d^{s}(w \square) .
$$

4. The $k$-termgale induced by a subprobability measure $p$ on $\{0,1\}^{*}$ is the family $d_{k}[p]=\left\{d_{k}^{s}[p] \mid\right.$ $s \in[0, \infty)\}$, where each $d_{k}^{s}[p]$ is defined by

$$
d_{k}^{s}[p](w)=2^{g_{k}(|w|, s)} \sum_{\substack{x \in\{0,1\}^{*} \\ w \sqsubseteq x \square}} p(x)
$$

for all $w \in \mathcal{T}$ with $|w|>m_{|k|}$.

Theorem 3.2. Let $k \in \mathbb{Z}$. If $\tilde{p}$ is an optimal constructive subprobability measure on $\{0,1\}^{*}$ then $d_{k}[\tilde{p}]$ is an optimal constructive $k$-termgale.

Proof. Similar to the proof of Theorem 5.4 in [26].

Corollary 3.3. For every $k \in \mathbb{Z}$, there exists an optimal constructive $k$-termgale.

Proof. It follows immediately from Theorems 2.1 and 3.2 .

Definition. Let $k \in \mathbb{Z}$ and $w \in\{0,1\}^{>m_{|k|}}$. Let $d_{k}$ be a constructive $k$-termgale. The scaled dimension of $w$ relative to $d_{k}$ is

$$
\operatorname{dim}_{d_{k}}(w)=\inf \left\{s \in[0, \infty) \mid d_{k}^{s}(w \square)>1\right\} .
$$

Notice that this definition depends on the constructive $k$-termgale chosen. The next two results prepare for a general definition of $k^{\text {th }}$ order scaled dimension of a string.

Proposition 3.4. Let $k \in \mathbb{Z}$. Let $\tilde{d}$ be an optimal constructive $k$-termgale and let $d$ be a constructive $k$-termgale. There exists $C>0$ such that

$$
\operatorname{dim}_{\tilde{d}}(w) \leq \operatorname{dim}_{d}(w)+\frac{C}{\frac{\partial g_{k}}{\partial s}(|w|+1,0)}
$$

for all $|w| \in\{0,1\}^{>m_{|k|}}$, where $\frac{\partial g_{k}}{\partial s}$ is the derivative of function $g_{k}(m, s)$ relative to its second argument.

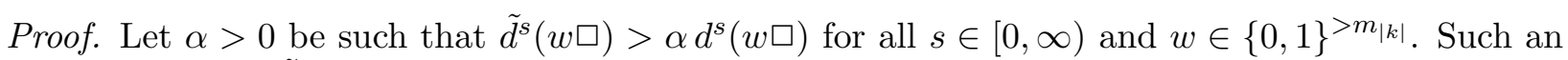
$\alpha$ exists because $\tilde{d}$ is an optimal constructive $k$-termgale.

Let $t \geq \operatorname{dim}_{d}(w)$. Define $h:\{0,1\}^{>m_{|k|}} \rightarrow[0, \infty)$ by $h(w)=s^{\prime}$, where $s^{\prime}$ is such that 


$$
g_{k}\left(|w|+1, t+s^{\prime}\right)-g_{k}(|w|+1, t)=\log \frac{1}{\alpha} .
$$

Notice that $h$ is well defined because for all $m \in \mathbb{N}$, the functions $g_{k}(m, \cdot):[0, \infty) \rightarrow \mathbb{R}$ are continuous and strictly increasing.

Let $s=t+h(w)$. For all $w \in\{0,1\}^{>m_{|k|}}$,

$$
\begin{aligned}
\tilde{d}_{k}^{s}(w \square)>\alpha d_{k}^{s}(w \square) & = \\
\alpha 2^{g_{k}(|w|+1, s)-g_{k}(|w|+1, t)} d_{k}^{t}(w \square) & \geq 1 .
\end{aligned}
$$

Thus,

$$
\operatorname{dim}_{\tilde{d}}(w) \leq s=t+h(w) \quad \forall w \in\{0,1\}^{>m_{|k|}} .
$$

Since $t$ can be chosen arbitrarily close to $\operatorname{dim}_{d}(w)$, for all $w \in\{0,1\}^{>m_{|k|}}$

$$
\operatorname{dim}_{\tilde{d}}(w) \leq \operatorname{dim}_{d}(w)+h(w) .
$$

The last part of the proof is to estimate the value of $h(w)$. By definition of $h$,

$$
g_{k}(|w|+1, t+h(w))-g_{k}(|w|+1, t)=\log \frac{1}{\alpha} .
$$

By the mean value theorem, there exist $\hat{s} \in(t, t+h(w))$ such that

$$
g_{k}(|w|+1, t+h(w))-g_{k}(|w|+1, t)=\frac{\partial g_{k}}{\partial s}(|w|+1, \hat{s}) h(w) .
$$

Then,

$$
h(w)=\frac{\log \frac{1}{\alpha}}{\frac{\partial g_{k}}{\partial s}(|w|+1, \hat{s})} \leq \frac{\log \frac{1}{\alpha}}{\frac{\partial g}{\partial s}(|w|+1,0)}
$$

where the last inequality holds since $\frac{\partial g_{k}}{\partial s}(m, \cdot)$ is an increasing function.

Corollary 3.5. Let $k \in \mathbb{Z}$ and let $\tilde{d}^{1}$ and $\tilde{d}^{2}$ be optimal constructive $k$-termgales. There exists a constant $C>0$ such that for all $w \in\{0,1\}^{>m_{|k|}}$,

$$
\left|\operatorname{dim}_{\tilde{d}^{1}}(w)-\operatorname{dim}_{\tilde{d}^{2}}(w)\right| \leq \frac{C}{\frac{\partial g_{k}}{\partial s}(|w|+1,0)} .
$$

It is easy to see that $\frac{\partial g_{k}}{\partial s}(m, 0) \rightarrow+\infty$ as $m \rightarrow \infty$. This means that if the definition of $k$ dimension is based on an optimal constructive $k$-termgale $\tilde{d}_{k}$, then the particular choice of $\tilde{d}_{k}$ has negligible impact on the dimension $\operatorname{dim}_{\tilde{d}_{k}}(w)$.

Definition. Fix $\tilde{d}_{k}$ an optimal constructive $k$-termgale. Let $k \in \mathbb{Z}$ and $w \in\{0,1\}^{>m_{|k|}}$. The $k^{\text {th }}$-order dimension of $w$ is

$$
\operatorname{dim}^{(k)}(w)=\operatorname{dim}_{\tilde{d}_{k}}(w)
$$


Remark. A natural question is the possibility to define resource-bounded scaled dimension of individual strings. The definition of scaled dimension of individual strings is based on the existence of an optimal constructive scaled termgale. This optimal termgale is defined using the optimal constructive subprobability measure $\mathbf{m}$. Unfortunately, there are no optimal subprobability measures in other interesting complexity classes of functions $\Delta$. Thus, the techniques that we use in this paper and previously in [26] cannot be applied to define $\Delta$-dimension of individual strings.

The optimal constructive subprobability measure $\mathbf{m}$ of a string $w$ is closely related to its Kolmogorov complexity [20, 21, 6]. This connection states that Kolmogorov complexity of a finite string is at most an additive constant away from the product of its length and its dimension [26]. In the case of scaled dimension of a string, we obtain a similar result: for every $k \in \mathbb{Z}$, the Kolmogorov complexity of a string is the scale function of order $k$ applied on the length of the string and the $k$-order scaled dimension.

Theorem 3.6. Let $k \in Z$. Then there exists a constant $c>0$ and $N \in \mathbb{N}$ such that

$$
\left|K(w)-g_{k}\left(|w|, \operatorname{dim}^{(k)}(w)\right)\right| \leq c \quad \forall w \in\{0,1\}^{>m_{|k|}} .
$$

Proof. Let $\mathbf{m}$ be the optimal constructive subprobability measure in Theorem 2.1. Let $c_{1}$ the constant in Theorem 2.2 such that for all $w \in\{0,1\}^{*}$,

$$
\left|K(w)-\log \frac{1}{\mathbf{m}(w)}\right| \leq c_{1}
$$

For all $w \in\{0,1\}^{>m_{|k|}}$ and $s \in[0, \infty)$,

$$
\begin{aligned}
d_{k}^{s}[\mathbf{m}](w \square)>1 & \Leftrightarrow 2^{g_{k}(|w|+1, s)} \mathbf{m}(w)>1 \\
& \Leftrightarrow g_{k}(|w|+1, s)>\log \frac{1}{\mathbf{m}(w)} .
\end{aligned}
$$

If $|w|$ is sufficiently large, $g_{k}(m, 0)>\log \frac{1}{\mathbf{m}(w)}$ and there exists $s_{w} \in[0, \infty)$ such that $g_{k}\left(|w|+1, s_{w}\right)=$ $\log \frac{1}{\mathbf{m}(w)}$. Then $\operatorname{dim}_{d_{k}[\mathbf{m}]}(w)=s_{w}$. So,

$$
\begin{aligned}
\left|K(w)-g_{k}\left(|w|, s_{w}\right)\right| & \leq\left|K(w)-g_{k}\left(|w|+1, s_{w}\right)\right|+\left|g_{k}\left(|w|+1, s_{w}\right)-g_{k}\left(|w|, s_{w}\right)\right| \\
& \leq\left|K(w)-\log \frac{1}{\mathbf{m}(w)}\right|+c_{2}<c_{1}+c_{2} .
\end{aligned}
$$

The next result states that the constructive scaled dimension of a sequence is characterized by the scaled dimension of its prefixes.

Theorem 3.7. Let $k \in \mathbb{Z}$ and $S \in C$,

$$
\operatorname{dim}^{(k)}(S)=\liminf _{n \rightarrow \infty} \operatorname{dim}^{(k)}(S[0 . . n-1]) .
$$

Proof. To see that $\operatorname{dim}^{(k)}(S) \leq \liminf _{n \rightarrow \infty} \operatorname{dim}^{(k)}(S[0 . . n-1])$, let $s$ and $s^{\prime}$ be rational numbers such that $s^{\prime}>s>\liminf _{n \rightarrow \infty} \operatorname{dim}^{(k)}(S[0 . . n-1])$. It suffices to show that $\operatorname{dim}^{(k)}(S) \leq s^{\prime}$. 
By our choice of s, there is an infinite set $J \subseteq \mathbb{N}$ such that for all $n \in J, \operatorname{dim}^{(k)}(S[0 . . n-1])<s$, whence $\tilde{d}_{k}^{s}(S[0 . . n-1])>1$. Define $d_{k}:\{0,1\}^{>m_{|k|}} \rightarrow[0, \infty)$ by

$$
d_{k}(w)=\frac{1}{2} \tilde{d}_{k}^{s^{\prime}}(w[0 . .|w|-2] \square)+\tilde{d}_{k}^{s^{\prime}}(w)
$$

Notice that $d_{k}$ is a constructive $s^{\prime(k)}$-supergale and for all $n \in J$,

$$
\begin{aligned}
d_{k}(S[0 . . n]) & =\frac{1}{2} \tilde{d}_{k}^{s^{\prime}}(S[0 . . n-1] \square)+\tilde{d}_{k}^{s^{\prime}}(S[0 . . n]) \\
& \geq \frac{1}{2} \tilde{d}_{k}^{s^{\prime}}(S[0 . . n-1] \square) \\
& =\frac{1}{2} 2^{g_{k}\left(n, s^{\prime}\right)-g_{k}(n, s)} \tilde{d}_{k}^{s}(S[0 . . n-1] \square) \\
& >\frac{1}{2} 2^{g_{k}\left(n, s^{\prime}\right)-g_{k}(n, s)}
\end{aligned}
$$

Since $J$ is infinite, this implies that $S \in S^{\infty}\left[d_{k}\right]$, whence $\operatorname{dim}^{(k)}(S) \leq s^{\prime}$.

To see that $\operatorname{dim}^{(k)}(S) \geq \liminf _{n \rightarrow \infty} \operatorname{dim}^{(k)}(S[0 . . n-1])$, let $s^{\prime}$ and $s$ be rational numbers such that $s^{\prime}>s>\operatorname{dim}^{(k)}(S)$. It suffices to show that there exist infinitely many $n \in \mathbb{N}$ for which $\operatorname{dim}^{(k)}(S[0 . . n-1]) \leq s^{\prime}$

Since $s>\operatorname{dim}^{(k)}(S)$, there is a constructive $s^{(k)}$-supergale $d_{k}$ such that $S \in S^{\infty}\left[d_{k}\right]$. Let $|w|>m_{|k|}$, define $d_{k}^{\prime}(w)=d_{k}(w)$ and $d_{k}^{\prime}(w \square)=\left[2^{\triangle g_{k}\left(|w|, s^{\prime}\right)}-2^{\triangle g_{k}(|w|, s)}\right] d_{k}(w)$. It is easy to check that $d_{k}^{\prime}$ is a constructive $s^{\prime(k)}$-supertermgale.

Let $s \in[0, \infty)$ and let $\widehat{d}_{k}^{s}: \mathcal{T} \rightarrow[0, \infty)$ defined by

$$
{\widehat{d_{k}}}^{s}(x)=2^{g_{k}(|x|, s)-g_{k}\left(|x|, s^{\prime}\right)} d_{k}^{\prime}(x)
$$

when $|x|>m_{|k|}$. The family ${\widehat{d_{k}}}=\left\{{\hat{d_{k}}}^{s} \mid s \in[0, \infty)\right\}$ is a constructive $k$-termgale. It follows by the

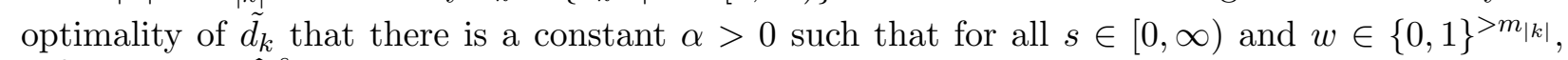
$\tilde{d}_{k}^{s}(w \square)>\alpha{\widehat{d_{k}}}^{s}(w \square)$. Since $S \in S^{\infty}\left[d_{k}\right]$ and $\Delta_{g_{k}}\left(n, s^{\prime}\right) \geq \Delta_{g_{k}}(n, s)$, there are infinitely many $n \in N$ such that

$$
\alpha\left[2^{\triangle g_{k}\left(n, s^{\prime}\right)}-2^{\triangle g_{k}(n, s)}\right] d_{k}((S[0 . . n-1])>1 .
$$

For all such $n$ we have

$$
\begin{aligned}
& \tilde{d}_{k}^{s^{\prime}}(S[0 . . n-1] \square)>\alpha{\widehat{d_{k}}}^{s^{\prime}}(S[0 . . n-1] \square)= \\
& \alpha\left[2^{\triangle g_{k}\left(n, s^{\prime}\right)}-2^{\triangle g_{k}(n, s)}\right] d_{k}(S[0 . . n-1])>1
\end{aligned}
$$

whence $\operatorname{dim}^{(k)}(S[0 . . n-1]) \leq s^{\prime}$.

\section{Scaled Dimension and Kolmogorov Complexity}

In this section we provide a characterization of scaled dimension in terms of Kolmogorov complexity.

Ryabko [28, 29], Staiger [30, 31], and Cai and Hartmanis [5] studied the relationship between Hausdorff dimension and Kolmogorov complexity, obtaining several results that are reviewed in $[26]$. 
With the development by Lutz of the constructive version of Hausdorff dimension it was possible to establish the following full characterization:

$$
\operatorname{cdim}(X)=\sup _{S \in X} \liminf _{n \rightarrow \infty} \frac{K(S[0 . . n-1])}{n} .
$$

This result was proved for individual infinite sequences by Mayordomo [27] in a direct way and also by Lutz [26] using the concept of dimension of finite sequences. Hitchcock [8] proved that space-bounded dimension and space-bounded Kolmogorov complexity are related in the same way:

$$
\operatorname{dim}_{\text {pspace }}(X)=\inf _{t \in \text { pspace }} \sup _{S \in X} \liminf _{n \rightarrow \infty} \frac{K S^{t}(S[0 . . n-1])}{n} .
$$

In this section, we extend all these results to scaled dimension. The following notation will be necessary in the characterization.

Definition. Let $S \in \mathbf{C}, k \in \mathbb{Z}$ and let $t: \mathbb{N} \rightarrow \mathbb{N}$ be a resource bound.

$$
\begin{aligned}
& \text { i) } \mathcal{K}_{(k)}(S)=\liminf _{n \rightarrow \infty} f_{k}(n, K(S[0 . . n-1]) . \\
& \text { ii) } \mathcal{K S}_{(k)}^{t}(S)=\liminf _{n \rightarrow \infty} f_{k}\left(n, K S^{t(n)}(S[0 . . n-1])\right) .
\end{aligned}
$$

where $f_{k}$ is defined in Section 2 as a partial inverse of $g_{k}$.

The following observation states the precise meaning of these concepts in terms of i.o. upper bounds.

Observation 4.1. Let $k \in \mathbb{Z}$ and $S \in \mathbf{C}$. Let $t$ be a resource bound. Then,

$$
\begin{aligned}
& \text { i) } \mathcal{K}_{(k)}(S)=\inf \left\{s \in[0, \infty) \mid \exists^{\infty} n K(S[0 . . n-1])<g_{k}(n, s)\right\} . \\
& \text { ii) } \mathcal{K S}_{(k)}^{t}(S)=\inf \left\{s \in[0, \infty) \mid \exists^{\infty} n K S^{t(n)}(S[0 . . n-1])<g_{k}(n, s)\right\} .
\end{aligned}
$$

For classes of languages we consider the worst-case upper bound.

Definition. Let $X \subseteq \mathbf{C}, k \in \mathbb{Z}$ and $j \in \mathbb{N}$,

$$
\begin{aligned}
\text { i) } & \mathcal{K}_{(k)}(X)=\sup _{S \in X} \mathcal{K}_{(k)}(S) . \\
\text { ii) } & \mathcal{K}_{(k)}^{\text {comp }}(X)=\inf _{t \in \operatorname{comp}} \sup _{S \in X} \mathcal{K} \mathcal{S}_{(k)}^{t}(S) . \\
\text { iii) } & \mathcal{K S}_{(k)}^{\mathrm{p}_{j} \text { space }}(X)=\inf _{t \in \mathrm{p}_{j} \text { space }} \sup _{S \in X} \mathcal{K} \mathcal{S}_{(k)}^{t}(S) .
\end{aligned}
$$

Notice that $\mathcal{K}_{(k)}^{\text {comp }}(X)$ can be also defined with the equivalent of $\mathcal{K S}_{(k)}^{t}(S)$ for time-bounded Kolmgorov complexity.

The main theorem of this section is the following characterization of scaled dimension.

Theorem 4.2. Let $X \subseteq \mathbf{C}$

1. For all $i, j \in \mathbb{N}$ with $i \leq j$,

$$
\begin{aligned}
\operatorname{dim}_{\mathrm{P}_{j} \text { space }}^{(i)}(X) & =\mathcal{K} \mathcal{S}_{(i)}^{\mathrm{p}_{j} \text { space }}(X), \\
\operatorname{dim}_{\mathrm{p}_{j} \text { space }}^{(-i)}(X) & =\mathcal{K} \mathcal{S}_{(-i)}^{\mathrm{p}_{j} \text { space }}(X) .
\end{aligned}
$$


2. For all $k \in \mathbb{Z}$,

$$
\begin{aligned}
\operatorname{cdim}^{(k)}(X) & =\mathcal{K}_{(k)}(X), \\
\operatorname{dim}_{\text {comp }}^{(k)}(X) & =\mathcal{K}_{(k)}^{\text {comp }}(X) .
\end{aligned}
$$

\section{Remarks.}

1. A similar characterization for the cases $\operatorname{dim}_{\mathrm{P}_{j} \text { space }}^{(-i)}(i>j)$ is not possible because it is known (Theorem 3.3 in [19]) that for each $S \in$ ESPACE there is an $\epsilon>0$ such that $K S^{2^{2 n}}\left(S_{\leq n}\right)<$ $2^{n+1}-2^{\epsilon n}$ a.e. $n$. Therefore,

$$
\mathcal{K S}_{(-2)}^{\text {pspace }}(\mathrm{ESPACE})=0
$$

whereas it is known that $\operatorname{dim}_{\text {pspace }}^{(-2)}(\mathrm{ESPACE})=1[25]$.

2. A dual version of Theorem 4.2 can be proven for the packing or strong dimension as characterized in [3].

\subsection{Proof of Theorem 4.2.}

We prove Theorem 4.2 from Lemmas 4.4 and 4.5. The first one states that dimension is smaller than $\mathcal{K}$ or $\mathcal{K S}$ (depending on the case) and it only holds for $i \leq j$ in the space-bounded case. The proof is based in the following scaled dimension version of the Borel-Cantelli Lemma [12].

Lemma 4.3. Let $k \in \mathbb{Z}$ and $s \in[0, \infty)$. If $d: \mathbb{N}^{2} \times\{0,1\}^{*} \rightarrow[0, \infty)$ is a $\Delta$-computable function such that for each $j, n \in \mathbb{N}, d_{j, n}$ is an $s^{(k)}$-gale, and such that for each $w$ with $|w|=m_{|k|}$ (remember that $m_{|k|}=\max \left\{a_{|k|}, 0\right\}$, where $\left(a_{|k|}, \infty\right)$ is the domain of $\left.g_{|k|}\right)$ the series

$$
\sum_{n=0}^{\infty} d_{j, n}(w)(j=0,1,2 \ldots),
$$

are uniformly $\Delta$-convergent, then

$$
\operatorname{dim}_{\Delta}^{(k)}\left(\bigcup_{j=0}^{\infty} \bigcap_{t=0}^{\infty} \bigcup_{n=t}^{\infty} S^{1}\left[d_{j, n}\right]\right) \leq s
$$

Lemma 4.4. Let $X \subseteq \mathbf{C}$

1. For all $i, j \in \mathbb{N}$ with $i \leq j$

$$
\begin{gathered}
\operatorname{dim}_{\mathrm{P}_{j} \text { space }}^{(i)}(X) \leq \mathcal{K} \mathcal{S}_{(i)}^{\mathrm{p}_{j} \text { space }}(X), \\
\operatorname{dim}_{\mathrm{p}_{j} \text { space }}^{(-i)}(X) \leq \mathcal{K} \mathcal{S}_{(-i)}^{\mathrm{p}_{j} \text { space }}(X) .
\end{gathered}
$$

2. For all $k \in \mathbb{Z}$

$$
\begin{gathered}
\operatorname{cdim}^{(k)}(X) \leq \mathcal{K}_{(k)}(X), \\
\operatorname{dim}_{\text {comp }}^{(k)}(X) \leq \mathcal{K}_{(k)}^{\text {comp }}(X) .
\end{gathered}
$$


Proof. To prove the first part of case 1 , let $s>s^{\prime}>\mathcal{K S}_{(i)}^{\mathrm{p}_{j} \text { space }}(X)$ be rational numbers. Let $t \in \mathrm{p}_{j}$ space with $t(n) \geq n$ such that

$$
\exists^{\infty} n K S^{t(n)}(S[0 . . n-1])<g_{i}\left(n, s^{\prime}\right)
$$

for all $S \in X$. Notice that such a $t$ exists by definition of $\mathcal{K S}_{(i)}^{\mathrm{p}_{j} \text { space }}(X)$ and Observation 4.1.

For all $n \in \mathbb{N}$, let

$$
Y_{n}=\left\{A \in \mathbf{C} \mid K S^{t(n)}(A[0 . . n-1])<g_{i}\left(n, s^{\prime}\right)\right\}
$$

Then

$$
X \subseteq \bigcap_{m=0}^{\infty} \bigcup_{n=m}^{\infty} Y_{n}
$$

Let $a \in[0, \infty)$ be such that $2 g_{i}\left(n, s^{\prime}\right)<g_{i}(n, s)+a$ for every $n \in \mathbb{N}$.

For all $n \in \mathbb{N}$ let $d_{n}:\{0,1\}^{*} \rightarrow[0, \infty)$ be defined by

$$
d_{n}(w)= \begin{cases}2^{-g_{i}\left(n, s^{\prime}\right)+a+g_{i}\left(m_{i}, s\right)} & \text { if }|w| \leq m_{i}, \\ 2^{g_{i}(|w|, s)-g_{i}\left(m_{i}, s\right)} d_{n}\left(w\left[0 . . m_{i}-1\right]\right) \rho(w) & \text { if } m_{i}<|w| \leq n, \\ 2^{g_{i}(|w|, s)-g_{i}(n, s)-|w|+n} d_{n}(w[0 . . n-1]) & \text { if }|w|>n,\end{cases}
$$

where

$$
\rho(w)=\frac{\#\left\{\pi|| \pi \mid<g_{i}\left(n, s^{\prime}\right), w \sqsubseteq U(\pi) \text { in } \leq t(n) \text { space }\right\}}{2^{g_{i}\left(n, s^{\prime}\right)}-1} .
$$

Then $d_{n}$ is an $s^{(i)}$-gale computable in $O(t(n))$-space for all $n \in \mathbb{N}$, and for all $w \in\{0,1\}^{m_{i}}$, the series $\sum_{n=0}^{\infty} d_{n}(w)$ is $\mathrm{p}_{j}$ space-convergent for $i \leq j$ (notice that this is not true for $i>j$ ). Moreover, for all $n \in \mathbb{N}, Y_{n} \subseteq S^{1}[d]$.

By Lemma $4.3, \operatorname{dim}_{\mathrm{P}_{j} \text { space }}^{(i)}(X) \leq s$. Since this holds for each $s>\mathcal{K S}_{(i)}^{\mathrm{p}_{j} \text { space }}(X)$ it follows that

$$
\operatorname{dim}_{\mathrm{p}_{j} \text { space }}^{(i)}(X) \leq \mathcal{K} \mathcal{S}_{(i)}^{\mathrm{p}_{j} \text { space }}(X)
$$

The proof of the second part of case 1 can be done in the same way by substituting $g_{i}$ by $g_{-i}$. The only change is in the definition of $d_{n}(w)$. In the case $|w| \leq m_{i}$ we must consider

$$
d_{n}(w)=2^{-g_{i}\left(n, s^{\prime}\right)+a+g_{-i}\left(m_{i}, s\right)} .
$$

The proof of case 2 is analogous and simpler since the resource-bounds do not need to be considered.

The second inequality ( $\mathcal{K}$ or $\mathcal{K} \mathcal{S}$ smaller than dimension) holds without restriction on the scale used. This will be useful in the next section.

Lemma 4.5. Let $X \subseteq$ C. For all $j \in \mathbb{N}, k \in \mathbb{Z}$

$$
\begin{gathered}
\mathcal{K S}_{(k)}^{\mathrm{p}_{j} \text { space }}(X) \leq \operatorname{dim}_{\mathrm{P}_{j} \text { space }}^{(k)}(X) \\
\mathcal{K}_{(k)}(X) \leq \operatorname{cdim}^{(k)}(X) \\
\mathcal{K}_{(k)}^{\text {comp }}(X) \leq \operatorname{dim}_{\text {comp }}^{(k)}(X)
\end{gathered}
$$


Proof. Let $s>\operatorname{dim}_{\mathrm{P}_{j} \text { space }}^{(k)}(X)$ and let $d$ be a $\mathrm{p}_{j}$ space-computable $s^{(k)}$-gale with $X \subseteq S^{\infty}[d]$. Let $t \in \mathrm{p}_{j}$ space be such that $t(n) \geq n$ and $d$ can be computed in space $t$. Assume without loss of generality that $d(w)<1$ for all $|w| \leq m_{|k|}$. By the inequality (Lemma 3.6 in [12]),

$$
\sum_{w \in\{0,1\}^{n}} d(w) \leq C 2^{g_{k}(n, s)},
$$

for all $n \geq m_{|k|}$ and $C=2^{-g_{k}\left(m_{|k|}, s\right)}$.

Define $A=\left\{w \in\{0,1\}^{*} \mid d(w)>1\right\}$. For all $n \geq m_{|k|},\left|A_{=n}\right|<C 2^{g_{k}(n, s)}$ and $A \in \mathrm{E}_{j}$ SPACE.

Each $w \in A_{=n}$ can be described by giving $n$ and its index within a list of $A_{=n}$ in lexicographical order. By reusing space, $w$ can be computed from this description in $3 t(n)$ space. Therefore, for all $w \in A_{=n}\left(n \geq m_{|k|}\right)$,

$$
K S^{3 t(n)}(w) \leq \log \left(\left|A_{=n}\right|\right)+O(\log n)<g_{k}(n, s)+O(\log n) .
$$

Let $S \in X$. Then

$$
\exists^{\infty} n \quad S[0 . . n-1] \in A_{=n}
$$

and

$$
\exists^{\infty} n K S^{3 t(n)}(S[0 . . n-1])<g_{k}(n, s)+O(\log n) .
$$

Therefore, $\mathcal{K S}_{(k)}^{3 t}(S) \leq s$ and $\mathcal{K} \mathcal{S}_{(k)}^{\mathrm{p}_{j} \text { space }}(X) \leq s$. Since this holds for each $s>\operatorname{dim}_{\mathrm{P}_{j} \text { space }}^{(k)}(X)$ it follows that $\mathcal{K S}_{(k)}^{\mathrm{p}_{j} \text { space }}(X) \leq \operatorname{dim}_{\mathrm{P}_{j} \text { space }}^{(k)}(X)$.

The proof of the second part is analogous.

Lemma 4.5 also holds in the case of polynomial time scaled dimension and the corresponding polynomial time-bounded Kolmogorov complexity. This can be proven by using the techniques proposed in [17] and [16]. The other inequality (Lemma 4.4) for polynomial time-bounds is a hard question given that reversible compression seems necessary to capture dimension [24].

Our characterization in Theorem 4.2 also holds when restricting to Kolmogorov complexity of prefixes of the form $A_{\leq n}$, except in the $0^{\text {th }}$-scale case.

Theorem 4.6. Let $X \subseteq \mathbf{C}$,

1. For all $i, j \in \mathbb{N}$ with $0<i \leq j$,

$$
\operatorname{dim}_{\mathrm{p}_{j} \text { space }}^{(i)}(X)<s
$$

iff there is a $t \in \mathrm{p}_{j}$ space such that for any $A \in X$

$$
K S^{t\left(2^{n+1}\right)}\left(A_{\leq n}\right)<g_{i}\left(2^{n+1}, s\right) \quad \text { i.o. n. }
$$

2. For all $i, j \in \mathbb{N}$ with $0<i \leq j$,

$$
\operatorname{dim}_{\mathrm{p}_{j} \text { space }}^{(-i)}(X)<s
$$

iff there is a $t \in \mathrm{p}_{j}$ space such that for any $A \in X$

$$
K S^{t\left(2^{n+1}\right)}\left(A_{\leq n}\right)<g_{-i}\left(2^{n+1}, s\right) \quad \text { i.o. } n .
$$

3. For all $k \in \mathbb{Z}, k \neq 0$,

$$
\operatorname{cdim}^{(k)}(X)<s
$$

iff for any $A \in X$

$$
K\left(A_{\leq n}\right)<g_{k}\left(2^{n+1}, s\right) \quad \text { i.o. } n .
$$


4. For all $k \in \mathbb{Z}, k \neq 0$,

$$
\operatorname{dim}_{\text {comp }}^{(k)}(X)<s
$$

iff there is a $t \in$ comp such that for any $A \in X$

$$
K T^{t\left(2^{n+1}\right)}\left(A_{\leq n}\right)<g_{k}\left(2^{n+1}, s\right) \quad \text { i.o. } n .
$$

Proof. $\Rightarrow$

Case 1. Let $s^{\prime}$ be a rational number such that $\operatorname{dim}_{\mathrm{p}_{j} \text { space }}^{(i)}(X)<s^{\prime}<s$. From Lemma 4.5 and Observation 4.1 there exists a $t \in \mathrm{p}_{j}$ space such that for every $A \in X$ and infinitely many $m$,

$$
K S^{t(m)}(A[0 . . m-1])<g_{i}\left(m, s^{\prime}\right) .
$$

Let $A \in X$ and let $m \in \mathbb{N}$ be such that (4.1) holds and $g_{i}\left(2 m, s^{\prime}\right)+\log (m+1) \leq g_{i}(m, s)$. Let $n$ be the largest natural number such that $2^{n+1}-1 \leq m . A\left[0 . .2^{n+1}-2\right]$ can be described by $A[0 . . m-1]$ and the removal of the remaining $m-2^{n+1}$ bits. Therefore,

$$
\begin{aligned}
K S^{t\left(2^{n+1}\right)+2^{n}}\left(A\left[0 . .2^{n+1}-2\right]\right) & <n+g_{i}\left(m, s^{\prime}\right) \\
& \leq \log (m+1)+g_{i}\left(m, s^{\prime}\right) \\
& \leq g_{i}\left(2^{n+1}, s\right)
\end{aligned}
$$

If $t^{\prime}(m)=t(m)+m / 2$ then $t^{\prime} \in \mathrm{p}_{j}$ space and the implication holds.

Case 2. Repeat the above argument but in this time let $m \in \mathbb{N}$ be such that

$$
K S^{t(m)}(A[0 . . m-1])<g_{-i}\left(m, 1-s^{\prime}\right) \leq m-g_{i}\left(m, 1-s^{\prime}\right)
$$

and $g_{i}(2 m, 1-s)+\log (m+1) \leq g_{i}\left(m, s^{\prime}\right)$. Let $n$ be the largest natural number such that $2^{n+1}-1 \geq$ $m$. Then, $A\left[0 . .2^{n+1}-2\right]$ can be described by $A[0 . . m-1]$ and adding the missing $2^{n+1}-m$ bits. In this case,

$$
\begin{aligned}
K S^{t\left(2^{n+1}\right)+2^{n}}\left(A\left[0 . .2^{n+1}-2\right]\right) & <n+m-g_{i}\left(m, 1-s^{\prime}\right)+\left(2^{n+1}-m\right) \\
& =\log (m+1)-g_{i}\left(m, 1-s^{\prime}\right)+2^{n+1} \\
& \leq 2^{n+1}-g_{i}\left(2^{n+1}, 1-s\right)=g_{-i}\left(2^{n+1}, s\right)
\end{aligned}
$$

Cases 3 and 4 . The proof is a combination of the other two cases.

$\Leftarrow$

For all cases, the proof follows directly from Observation 4.1 and Lemma 4.4.

For example, $\operatorname{dim}_{\text {pspace }}^{(-1)}(X)<s$ iff there is a $c$ such that for any $A \in X$

$$
K S^{2^{c(n+1)}}\left(A_{\leq n}\right)<2^{n+1}-2^{(n+1)(1-s)} \text { i.o. } n .
$$

Remark. Notice that it is not equivalent in general to consider $K S\left(A_{=n}\right)$ and $K S\left(A_{\leq n}\right)$. Whereas $K S^{2^{c n}}\left(A_{=n}\right)<2^{n}-2^{\epsilon n}$ implies that $K S^{2^{c^{\prime} n}}\left(A_{\leq n}\right)<2^{n+1}-2^{\epsilon n}$, the quantity $K S^{2^{c n}}\left(A_{\leq n}\right)$ can be much lower than $K S\left(A_{=n}\right)$, relative to the corresponding length. Juedes and Lutz extensively studied $K S\left(A_{=n}\right)$ in [19], mainly for languages in ESPACE and languages that are $\leq_{\mathrm{m}}^{\mathrm{P} / \text { poly }}$-hard, that is, hard for many-one non-uniform reductions. Theorem 4.6 will be used in Section 5 to study $K S\left(A_{\leq n}\right)$ for $\leq_{\mathrm{T}}^{\mathrm{P} / \text { poly }}$-hard languages in ESPACE. 


\subsection{Alternative proof for constructive scaled dimension}

Here we present an alternative proof of the characterization for constructive scaled dimension that is a corollary of Theorems 3.6 and 3.7.

Corollary 4.7. Let $X \subseteq \mathbf{C}$ and $k \in \mathbb{Z}$,

$$
\operatorname{cdim}^{(k)}(X)=\mathcal{K}_{(k)}(X) .
$$

Proof. It was proved by Lutz [26] that

$$
\operatorname{cdim}(X)=\sup _{S \in X} \operatorname{dim}(S),
$$

and therefore it is enough to show that $\operatorname{dim}(S)=\mathcal{K}_{(k)}(S)$ for each sequence $S$.

By Theorem 3.6, there exist $c>0$ and $N \in \mathbb{N}$ such that

$$
\left|K(w)-g_{k}\left(|w|, s_{w}\right)\right|<c \quad \forall w \in\{0,1\}^{>m_{|k|}},
$$

where $s_{w}=\operatorname{dim}^{(k)}(w)$.

On the other hand, for all $|w|>m_{|k|}$, since $f_{k}$ is the inverse of $g_{k}$,

$$
K(w)=g_{k}\left(|w|, f_{k}(|w|, K(w))\right)
$$

By the mean value theorem there exists $s_{w}^{\prime}$ such that

$$
\min \left\{f_{k}(|w|, K(w)), s_{w}\right\} \leq s_{w}^{\prime} \leq \max \left\{f_{k}(|w|, K(w)), s_{w}\right\}
$$

and

$$
\begin{gathered}
\left|g_{k}\left(|w|, f_{k}(|w|, K(w))\right)-g_{k}\left(|w|, s_{w}\right)\right|= \\
\frac{\partial g_{k}}{\partial s}\left(|w|, s_{w}^{\prime}\right)\left|f_{k}(|w|, K(w))-s_{w}\right| .
\end{gathered}
$$

By (4.2), (4.3) and (4.4)

$$
\left|f_{k}(|w|, K(w))-s_{w}\right|<\frac{c}{\frac{\partial g_{k}}{\partial s}(|w|, 0)},
$$

where $s_{w}^{\prime}$ can be replaced by 0 because $\frac{\partial g_{k}}{\partial s}(m,$.$) is an increasing function for all m \in \mathbb{N}$. Then, by Theorem 3.7,

$$
\operatorname{dim}^{(k)}(S)=\liminf _{n \rightarrow \infty} \operatorname{dim}^{(k)}(S[0 . . n-1])=\liminf _{n \rightarrow \infty} f_{k}(n, K(S[0 . . n-1]))
$$

where the last equality holds by applying (4.5) in $S[0 . . n-1]$ and using the fact that

$$
\lim _{n \rightarrow \infty} \frac{c}{\frac{\partial g_{k}}{\partial s}(n, 0)}=0
$$




\section{Small Spans and the Kolmogorov Complexity of Hard Sets}

In this section we study the behavior of $\mathrm{P} /$ poly-Turing reductions in the class ESPACE. These reductions, denoted by $\leq_{\mathrm{T}}^{\mathrm{P} / \text { poly }}$, are Turing reductions which are computed by a nonuniform family of polynomial-size circuits.

The lower and upper spans of a set are defined as follows.

Definition. Let $A \subseteq\{0,1\}^{*}$.

1. The $\leq_{\mathrm{T}}^{\mathrm{P} / \text { poly }}$ - lower span of $\mathrm{A}$ is

$$
(\mathrm{P} / \text { poly })_{\mathrm{T}}(A)=\left\{B \subseteq\{0,1\}^{*} \mid B \leq_{\mathrm{T}}^{\mathrm{P} / \text { poly }} A\right\} .
$$

2. The $\leq_{\mathrm{T}}^{\mathrm{P} / \text { poly }}$ - upper span of $\mathrm{A}$ is

$$
(\mathrm{P} / \text { poly })_{\mathrm{T}}^{-1}(A)=\left\{B \subseteq\{0,1\}^{*} \mid A \leq_{\mathrm{T}}^{\mathrm{P} / \text { poly }} B\right\} .
$$

Juedes and Lutz proved the following small span theorem for these reductions.

Theorem 5.1. (Juedes and Lutz [19]) For every $A \in$ ESPACE.

$$
\mu\left((\mathrm{P} / \text { poly })_{\mathrm{T}}(A) \mid \mathrm{ESPACE}\right)=0
$$

or

$$
\mu_{\text {pspace }}\left((\mathrm{P} / \text { poly })_{\mathrm{T}}^{-1}(A)\right)=0 .
$$

This theorem states that for each $A \in$ ESPACE, at least one of the lower and upper spans of $A$ is small in the sense of resource-bounded measure. Small span theorems for the class of exponential time languages and polynomial time reductions have been studied for both measure and dimension $[18,1,23,2,4,10]$. Here we prove the following strengthening of Theorem 5.1.

Theorem 5.2. For every $A \in \mathrm{ESPACE}$,

$$
\operatorname{dim}^{(1)}\left((\mathrm{P} / \operatorname{poly})_{\mathrm{T}}(A) \mid \mathrm{ESPACE}\right)=0
$$

or

$$
\operatorname{dim}_{\text {pspace }}^{(-3)}\left((\mathrm{P} / \text { poly })_{\mathrm{T}}^{-1}(A)\right)=0
$$

Proof. Let

$$
Z=\left\{A \subseteq\{0,1\}^{*} \mid \operatorname{dim}_{\text {pspace }}^{(-3)}\left((\mathrm{P} / \text { poly })_{\mathrm{T}}^{-1}(A)\right)>0\right\} .
$$

It is enough to show that

$$
\operatorname{dim}^{(1)}(Z \mid \mathrm{ESPACE})=0 .
$$

Given (5.1), we consider two cases:

1. $(\mathrm{P} / \text { poly })_{\mathrm{T}}(A) \cap \mathrm{ESPACE} \subseteq Z$.

Then it follows that

$$
\operatorname{dim}^{(1)}\left((\mathrm{P} / \text { poly })_{\mathrm{T}}(A) \mid \mathrm{ESPACE}\right) \leq \operatorname{dim}^{(1)}(Z \mid \mathrm{ESPACE})=0 .
$$


2. $(\mathrm{P} / \text { poly })_{\mathrm{T}}(A) \cap \mathrm{ESPACE} \nsubseteq \nsubseteq Z$.

Then there is a language $B \in(\mathrm{P} / \text { poly })_{\mathrm{T}}(A) \cap$ ESPACE such that $B \notin Z$. Because $B \leq_{\mathrm{T}}^{\mathrm{P} / \text { poly }} A$, we have $(\mathrm{P} / \text { poly })_{\mathrm{T}}^{-1}(A) \subseteq(\mathrm{P} / \text { poly })_{\mathrm{T}}^{-1}(B)$ and therefore

$$
\operatorname{dim}_{\mathrm{pspace}}^{(-3)}\left((\mathrm{P} / \text { poly })_{\mathrm{T}}^{-1}(A)\right) \leq \operatorname{dim}_{\mathrm{pspace}}^{(-3)}\left((\mathrm{P} / \text { poly })_{\mathrm{T}}^{-1}(B)\right)
$$

by the monotonicity of scaled dimension. Because $B \notin Z$, we have $\operatorname{dim}_{\mathrm{pspace}}^{(-3)}\left((\mathrm{P} / \text { poly })_{\mathrm{T}}^{-1}(B)\right)=$ 0 , so $\operatorname{dim}_{\text {pspace }}^{(-3)}\left((\mathrm{P} / \text { poly })_{\mathrm{T}}^{-1}(B)\right)=0$ follows.

Our proof of (5.1) is based on the proof of Theorem 4.5 in [19]. We begin by recalling some of the definitions and notations from that proof, slightly adapting them for use in this proof.

For each $r \in \mathbb{N}$, define the functions $a_{r}, b_{r}: \mathbb{N} \rightarrow \mathbb{N}$ by

$$
a_{r}(n)=n^{r}+r \quad \text { and } \quad b_{r}(n)=\sum_{i=0}^{n} a_{r}(i) .
$$

Let $\mathrm{ADV}_{r}$ be the class of all advice functions $h: \mathbb{N} \rightarrow\{0,1\}^{*}$ satisfying $|h(n)|=a_{r}(n)$ for all $n \in \mathbb{N}$. For any $A, B \subseteq\{0,1\}^{*}$ satisfying $A \leq_{\mathrm{T}}^{\mathrm{P} / \text { poly }} B$, there exist $r, k \in \mathbb{N}$ and $h \in \mathrm{ADV}_{r}$ such that

$$
A=L\left(M_{k}^{B} / h\right) \text {, }
$$

where $M_{k}$ is the $k^{\text {th }}$ polynomial time-bounded oracle Turing machine.

A partial $a_{r}(n)$-advice function is a finite function

$$
h^{\prime}:\{0,1, \ldots, k-1\} \rightarrow\{0,1\}^{*}
$$

for some $k \in \mathbb{N}$, such that for all $0 \leq n<k,\left|h^{\prime}(n)\right|=a_{r}(n)$. For each partial $a_{r}(n)$-advice function $h^{\prime}$, the cylinder generated by $h^{\prime}$ is

$$
\mathrm{CYL}\left(h^{\prime}\right)=\left\{h \in \mathrm{ADV}_{r} \mid h\left\lceil\{0,1, \ldots, k-1\}=h^{\prime}\right\},\right.
$$

where $h\lceil\{0,1, \ldots, k-1\}$ denotes $h$ restricted to domain $\{0,1, \ldots, k-1\}$. The probability of this cylinder is defined to be

$$
\operatorname{Pr}\left(\operatorname{CYL}\left(h^{\prime}\right)\right)=\prod_{n=0}^{k-1} 2^{-a_{r}(n)} .
$$

For each $r \in \mathbb{N}$, we will use the sample space

$$
\Omega_{r}=\mathrm{ADV}_{r} \times \mathbf{C} .
$$

Here we use the product probability measure, with the above probability measure on $\mathrm{ADV}_{r}$ and the uniform distribution on $\mathbf{C}$. For each $r, k, j \in \mathbb{N}$, define the event $\mathcal{E}_{r, k, j}^{A} \subseteq \Omega_{r}$ by

$$
\mathcal{E}_{r, k, j}^{A}=\left\{(h, B) \mid(\forall 0 \leq i<j) \llbracket s_{i} \in A \rrbracket=\llbracket s_{i} \in L\left(M_{k}^{B} / h\right) \rrbracket\right\} .
$$

For each $r, k, j \in \mathbb{N}$ and $A \subseteq\{0,1\}^{*}$, let

$$
N_{A}(r, k, j)=\left|\left\{i<j \mid \operatorname{Pr}\left(\mathcal{E}_{r, k, i+1}^{A}\right) \leq \frac{1}{2} \operatorname{Pr}\left(\mathcal{E}_{r, k, i}^{A}\right)\right\}\right| .
$$


Then for all $r, k, j \in \mathbb{N}$ and $A \subseteq\{0,1\}^{*}$, we have

$$
\operatorname{Pr}\left(\mathcal{E}_{r, k, j}^{A}\right) \leq 2^{-N_{A}(r, k, j)} .
$$

For each $A \subseteq\{0,1\}^{*}$ and rational numbers $s, \delta>0$, define an $s^{(-3)}$-gale $d_{s, \delta}^{A}:\{0,1\}^{*} \rightarrow[0, \infty)$ by

$$
d_{s, \delta}^{A}(w)=2^{-g_{3}(|w|, 1-s)} \sum_{r=0}^{\infty} \sum_{k=0}^{\infty} \sum_{j=0}^{\infty} 2^{-(r+k) / 4-j^{\delta}} \cdot d_{r, k, j}^{A}(w),
$$

where for all $r, k, j \in \mathbb{N}, d_{r, k, j}^{A}$ is the martingale

$$
d_{r, k, j}^{A}(w)= \begin{cases}2^{|w|} \operatorname{Pr}\left(\mathrm{ADV}_{r} \times \mathbf{C}_{w} \mid \mathcal{E}_{r, k, j}^{A}\right) & \text { if } \operatorname{Pr}\left(\mathcal{E}_{r, k, j}^{A}\right)>0 \\ 1 & \text { if } \operatorname{Pr}\left(\mathcal{E}_{r, k, j}^{A}\right)=0 .\end{cases}
$$

It is routine to show that $d_{s, \delta}^{A}$ is pspace-computable if $A \in$ ESPACE.

Let $A, B \subseteq\{0,1\}^{*}, k, r \in \mathbb{N}$, and $h \in \mathrm{ADV}_{r}$ such that $A=L\left(M_{k}^{B} / h\right)$. There is a polynomial time-bound on $M_{k}$ and a polynomial length bound on $h$, so there is a constant $c \in \mathbb{N}$ so that all queries of $\left(M_{k}^{B} / h\right)\left(s_{i}\right)$ have length strictly bounded by $\left|s_{i}\right|^{c}$ for all sufficiently large $i$. Defining $n(i)=\lceil\log (i+2)-1\rceil$, we have $\left|s_{i}\right|=n(i)$ for all $i$. For now, fix $j \in \mathbb{N}$. If we choose

$$
l=2^{(\log (j+1))^{c}},
$$

then all queries of $L\left(M_{k}^{B} / h\right)\left(s_{i}\right)$ for $0 \leq i<j$ are among $s_{0}, s_{1}, \ldots, s_{l-1}$. In other words, $A[0 . . j-1]$ is determined by $B[0 . . l-1]$. Note that

$$
j+1=2^{(\log l)^{\frac{1}{c}}} .
$$

Let $h_{j}=h\left\lceil\{0,1, \ldots, n(j-1)\}\right.$. Then $h_{j}$ is a restriction of $h$ that provides advice for all the inputs $s_{0}, \ldots, s_{j-1}$. It follows that $\mathrm{CYL}\left(h_{j}\right) \times \mathbf{C}_{B[0 . . l-1]} \subseteq \mathcal{E}_{r, k, j}^{A}$, so we can argue as in [19] that

$$
\operatorname{Pr}\left(\mathcal{E}_{r, k, j}^{A} \mid \mathrm{ADV}_{r} \times \mathbf{C}_{B[0 . l-1]}\right) \geq 2^{-b_{r}(n(j))},
$$

and then obtain

$$
d_{r, k, j}^{A}(B[0 . . l-1]) \geq 2^{N_{A}(r, k, j)-b_{r}(n(j))} .
$$

Let $\epsilon>\delta>0$ and define

$$
X_{\epsilon}=\left\{A \subseteq\{0,1\}^{*} \mid(\forall k)(\forall r)\left(\forall^{\infty} j\right) N_{A}(r, k, j)>j^{\epsilon}\right\} .
$$

We claim that $X_{\epsilon} \cap \mathrm{ESPACE} \subseteq Z^{c}$, i.e., that

$$
\operatorname{dim}_{\text {pspace }}^{(-3)}\left((\mathrm{P} / \text { poly })_{\mathrm{T}}^{-1}(A)\right)=0
$$

for every $A \in X_{\epsilon} \cap$ ESPACE. For this, let $A \in X_{\epsilon} \cap \mathrm{ESPACE}$ and let $B \in(\mathrm{P} / \operatorname{poly})_{\mathrm{T}}^{-1}(A)$. Then there exist $k, r \in \mathbb{N}$ and $h \in \mathrm{ADV}_{r}$ such that $A=L\left(M_{k}^{B} / h\right)$. Let $j$ be sufficiently large to ensure $N_{A}(r, k, j)>j^{\epsilon}$. Then, defining $c$ and $l$ as above, we have

$$
\begin{aligned}
\log d_{s, \delta}^{A}(B[0 . . l-1]) & \geq \log d_{r, k, j}^{A}(B[0 . . l-1])-g_{3}(l, 1-s)-(r+k) / 4-j^{\delta} \\
& \geq j^{\epsilon}-b_{r}(n(j))-g_{3}(l, 1-s)-(r+k) / 4-j^{\delta} \\
& =\left(2^{(\log l)^{1 / c}}-1\right)^{\epsilon}-b_{r}(n(j))-2^{2^{(\log \log l)^{(1-s)}}}-(r+k) / 4-\left(2^{(\log l)^{1 / c}}-1\right)^{\delta} .
\end{aligned}
$$


Since $r$ and $k$ are constants here, it follows that $B \in S^{\infty}\left[d_{s, \delta}^{A}\right]$. Therefore $(\mathrm{P} / \text { poly })_{\mathrm{T}}^{-1}(A) \subseteq S^{\infty}\left[d_{s, \delta}^{A}\right]$. Since $A \in \mathrm{ESPACE}, d_{s, \delta}^{A}$ is pspace-computable, so $\operatorname{dim}_{\mathrm{pspace}}^{(-3)}\left((\mathrm{P} / \mathrm{poly})_{\mathrm{T}}^{-1}(A)\right) \leq s$. This holds for all $s>0$, so we obtain (5.2).

Now we show that for every $\epsilon>0$,

$$
\operatorname{dim}_{\text {pspace }}^{(1)}\left(X_{\epsilon}^{c}\right) \leq \epsilon .
$$

Let $A \in X_{\epsilon}^{c}$. Then there exist $r, k \in \mathbb{N}$ such that $N_{A}(r, k, j) \leq j^{\epsilon}$ for infinitely many $j \in \mathbb{N}$. Notice that $N_{A}(r, k, j)$ is determined by $A[0 . . j-1]$. For each $j \in \mathbb{N}$, let

$$
Z_{r, k, j}=\left\{B[0 . . j-1] \mid N_{B}(r, k, j) \leq j^{\epsilon}\right\} \subseteq\{0,1\}^{j} .
$$

We can bound the size of $Z_{r, k, j}$ as

$$
\left|Z_{r, k, j}\right| \leq j^{\epsilon}\left(\begin{array}{l}
j \\
j^{\epsilon}
\end{array}\right) 2^{j^{\epsilon}} \leq j^{\epsilon} \cdot 2^{\mathcal{H}\left(j^{\epsilon-1}\right) j+j^{\epsilon}}
$$

because we can specify an element of the set by first identifying the at most $j^{\epsilon}$ positions $i$ on which $\mathcal{E}_{r, k, i+1}^{A} \leq \frac{1}{2} \mathcal{E}_{r, k, i}^{A}$ and then using $j^{\epsilon}$ bits to specify which of the two possibilities to use for the $i^{\text {th }}$ bit in case $\mathcal{E}_{r, k, i+1}^{A}=\frac{1}{2} \mathcal{E}_{r, k, i}^{A}$. Therefore

$$
\mathcal{H}\left(j^{\epsilon-1}\right) j+j^{\epsilon}+\log j
$$

bits are enough to identify each string in $Z_{r, k, j}$, where $\mathcal{H}(x)$ is the binary entropy $\mathcal{H}(x)=x \log x+$ $(1-x) \log (1-x)$. From this description along with encodings of $r, k$, and $j$ we can compute the string using polynomial space: for some polynomial $p$ we have

$$
K S^{p}(w) \leq \mathcal{H}\left(j^{\epsilon-1}\right) j+j^{\epsilon}+2 \log j+\log r+\log k
$$

for all $w \in Z_{r, k, j}$. We have a single polynomial $p$ that works for every $r, k$ and for every $j \geq j_{0}(r, k)$ for some $j_{0}(r, k)$.

Notice that

$$
\begin{aligned}
\mathcal{H}\left(j^{\epsilon-1}\right) j & =\left(j^{\epsilon-1} \log j^{1-\epsilon}+\left(1-j^{\epsilon-1}\right) \log \frac{1}{1-j^{\epsilon-1}}\right) j \\
& =j^{\epsilon}(1-\epsilon) \log j+j\left(1-j^{\epsilon-1}\right) \log \left(1+\frac{j^{\epsilon-1}}{1-j^{\epsilon-1}}\right) \\
& \leq j^{\epsilon}(1-\epsilon) \log j+j\left(1-j^{\epsilon-1}\right) \frac{j^{\epsilon-1}}{1-j^{\epsilon-1}} \log e \\
& =j^{\epsilon}[(1-\epsilon) \log j+\log e] .
\end{aligned}
$$

It follows from the above that $\mathcal{K S}_{(1)}^{p}(A) \leq \epsilon$ because $A$ satisfies $A[0 . . j-1] \in Z_{r, k, j}$ infinitely often. Since $A \in X_{\epsilon}$ is arbitrary and the polynomial $p$ does not depend on $A$, we have $\mathcal{K S}_{(1)}^{\text {pspace }}\left(X_{\epsilon}\right) \leq \epsilon$. Appealing to Theorem 4.2, we establish (5.3).

We proved that $X_{\epsilon} \cap \operatorname{ESPACE} \subseteq Z^{c}$ for all $\epsilon \in(0,1)$. This implies $Y^{c} \cap \operatorname{ESPACE} \subseteq X_{\epsilon}^{c}$, so

$$
\operatorname{dim}^{(1)}(Z \mid \mathrm{ESPACE})=\operatorname{dim}_{\text {pspace }}^{(1)}(Z \cap \operatorname{ESPACE}) \leq \operatorname{dim}_{\text {pspace }}^{(1)}\left(X_{\epsilon}^{c}\right) \leq \epsilon
$$

for all $\epsilon \in(0,1)$. Therefore $\operatorname{dim}^{(1)}(Z \mid \mathrm{ESPACE})=0$. 
Theorem 5.2 improves Theorem 5.1 because $\operatorname{dim}^{(-3)}(X)<1$ implies $\mu_{\text {pspace }}(X)=0$. Also, in [10] it is shown that $(-2)^{\text {nd }}$-scaled small span theorems are not possible, since for $A \mathrm{a} \leq_{\mathrm{m}}^{\mathrm{P}}$-complete language for ESPACE, $\operatorname{dim}_{\mathrm{pspace}}^{(-2)}\left(\mathrm{P}_{\mathrm{m}}^{-1}(A)\right)=1$. Therefore we can't substitute -3 by a bigger scale in the statement of Theorem 5.2.

Because of the connections we have obtained between scaled dimension and Kolmogorov complexity we can conclude the following.

Theorem 5.3. For every $A \in \mathrm{ESPACE}$, if

$$
\operatorname{dim}^{(1)}\left((\mathrm{P} / \text { poly })_{\mathrm{T}}(A) \mid \mathrm{ESPACE}\right)>0
$$

then

$$
\mathcal{K S}_{\text {pspace }}^{(-3)}\left((\mathrm{P} / \text { poly })_{\mathrm{T}}^{-1}(A)\right)=0
$$

Proof. The theorem follows from Theorem 5.2 and Lemma 4.5.

In particular for hard languages we have the following corollary.

Corollary 5.4. Let $\mathcal{H}$ be the class of languages that are $\leq_{\mathrm{T}}^{\mathrm{P} / \text { poly }}$-hard for ESPACE. Then

$$
\mathcal{K S}_{\text {pspace }}^{(-3)}(\mathcal{H})=0 .
$$

That is, for each $\epsilon>0$ there is a c such that for every $\leq_{\mathrm{T}}^{\mathrm{P} / \text { poly }}$-hard language $H$,

$$
K S^{2^{c n}}\left(H_{\leq n}\right)<2^{n+1}-2^{2^{(\log n)^{1-\epsilon}}} \text { i.o. n. }
$$

In fact, by examining the proof of Theorem 5.2, we obtain a stronger bound in the following theorem. This matches the upper bound given by Juedes and Lutz [19] for the $\leq_{\mathrm{m}}^{\mathrm{P} / \mathrm{poly}}$-hard languages.

Theorem 5.5. There is a constant $c$ such that for every $\leq_{\mathrm{T}}^{\mathrm{P} / \text { poly }}$-hard language $H$ for ESPACE, there is some $\epsilon>0$ such that

$$
K S^{2^{c n}}\left(H_{\leq n}\right)<2^{n+1}-2^{n^{\epsilon}} \text { i.o. } n .
$$

Proof. Let $\epsilon=\frac{1}{2}$ and recall the set $X_{\epsilon}$ in the proof of Theorem 5.2. Since $\operatorname{dim}_{\text {pspace }}^{(1)}(\mathrm{ESPACE})=1$, we have ESPACE $\nsubseteq X_{\epsilon}^{c}$. Let $A \in \operatorname{ESPACE} \cap X_{\epsilon}^{c}$.

Let $B$ be $\leq_{\mathrm{T}}^{\mathrm{P} / \text { poly }}$-hard for ESPACE. Then $A$ reduces to $B$ via some $\leq_{\mathrm{T}}^{\mathrm{P} / \text { poly }}$-reduction. Choose $c$ so that for all sufficiently large $i$, all queries of this reduction on input $s_{i}$ have length strictly bounded by $\left|s_{i}\right|^{c}$. The proof of (5.2) shows that the $s^{(-3)}$-gale $d_{s, \delta}^{A}$ succeeds on $B$.

Let $\gamma \in\left(1-\frac{\epsilon}{c}, 1\right)$. Define a $\gamma^{(-2)}$-gale $d$ by

$$
d(w)=2^{g_{3}(|w|, 1-s)-g_{2}(|w|, 1-\gamma)} d_{s, \delta}^{A}(w) .
$$

Then the calculation showing that $d_{s, \delta}^{A}$ succeeds on $B$ changes to

$$
\log d(B[0 . . l-1]) \geq\left(2^{(\log l)^{1 / c}}-1\right)^{\epsilon}-b_{r}(n(j))-2^{(\log l)^{1-\gamma}}-(r+k) / 4-\left(2^{(\log l)^{1 / c}}-1\right)^{\delta} .
$$

Because $1-\gamma<\frac{\epsilon}{c}, d$ also succeeds on $B$. Therefore $\operatorname{dim}_{\text {pspace }}^{(-2)}(H) \leq \gamma<1$.

Let $\alpha \in(\gamma, 1)$. From Lemma 4.5 (as applied in Theorem 4.6) we obtain that

$$
K S^{2^{d n}}\left(B_{\leq n}\right)<2^{n+1}-2^{n^{1-\alpha}}
$$

for infinitely many $n$. Here $d$ is a constant that does not depend on $B$. 
This result tells us that the $\leq_{\mathrm{T}}^{\mathrm{P} / \text { poly }}$-hard languages are unusually simple, since for most languages the opposite holds, even when allowing any resource bound on the Kolmogorov complexity.

Theorem 5.6. For every resource bound t, the class of all sets $A$ that satisfy

$$
K S^{t\left(2^{n}\right)}\left(A_{\leq n}\right)<2^{n+1}-2^{n^{\epsilon}} \text { i.o. } n
$$

for some $\epsilon>0$ has $(-3)^{\text {rd }}$-order comp-dimension 0 .

Proof. The result follows from our characterization in Theorem 4.2.

Theorem 5.6 implies that most decidable languages (in a very strong sense) satisfy the lower bound

$$
K S^{2^{c n}}\left(A_{\leq n}\right) \geq 2^{n+1}-2^{n^{\epsilon}} \text { a.e. } n
$$

for every $\epsilon>0$, whereas $\leq_{\mathrm{T}}^{\mathrm{P} / \text { poly }}$-hard sets have the opposite property by Theorem 5.5. In fact the best known lower bound for $\leq_{\mathrm{T}}^{\mathrm{P} / \text { poly }}$-hard sets is much lower, in [19] it is proven that for each $\leq_{\mathrm{T}}^{\mathrm{P} / \text { poly }}$-hard $H$ there is an $\epsilon>0$ such that

$$
K S^{2^{n^{\epsilon}}}\left(H_{\leq n}\right)>2^{n^{\epsilon}} \text { a.e. } n \text {. }
$$

\section{Acknowledgement}

We thank Jack Lutz for very helpful discussions on reference [19] and an anonymous referee for many helpful suggestions.

\section{References}

[1] K. Ambos-Spies, W. Merkle, J. Reimann, and F. Stephan. Hausdorff dimension in exponential time. In Proceedings of the 16th IEEE Conference on Computational Complexity, pages 210 $217,2001$.

[2] K. Ambos-Spies, H.-C. Neis, and S. A. Terwijn. Genericity and measure for exponential time. Theoretical Computer Science, 168(1):3-19, 1996.

[3] K. B. Athreya, J. M. Hitchcock, J. H. Lutz, and E. Mayordomo. Effective strong dimension in algorithmic information and computational complexity. SIAM Journal on Computing. To appear.

[4] H. Buhrman and D. van Melkebeek. Hard sets are hard to find. Journal of Computer and System Sciences, 59(2):327-345, 1999.

[5] J. Cai and J. Hartmanis. On Hausdorff and topological dimensions of the Kolmogorov complexity of the real line. Journal of Computer and Systems Sciences, 49:605-619, 1994.

[6] G. J. Chaitin. A theory of program size formally identical to information theory. Journal of the Association for Computing Machinery, 22:329-340, 1975.

[7] X. Gu. A note on dimensions of polynomial size circuits. Theoretical Computer Science, $359(1-3): 176-187,2006$. 
[8] J. M. Hitchcock. Effective Fractal Dimension: Foundations and Applications. PhD thesis, Iowa State University, 2003.

[9] J. M. Hitchcock. Gales suffice for constructive dimension. Information Processing Letters, 86(1):9-12, 2003.

[10] J. M. Hitchcock. Small spans in scaled dimension. SIAM Journal on Computing, 34(1):170194, 2004.

[11] J. M. Hitchcock. Hausdorff dimension and oracle constructions. Theoretical Computer Science, 355(3):382-388, 2006.

[12] J. M. Hitchcock, J. H. Lutz, and E. Mayordomo. Scaled dimension and nonuniform complexity. Journal of Computer and System Sciences, 69:97-122, 2004.

[13] J. M. Hitchcock, J. H. Lutz, and E. Mayordomo. The fractal geometry of complexity classes. SIGACT News, 36(3):24-38, September 2005.

[14] J. M. Hitchcock and A. Pavan. Hardness hypotheses, derandomization, and circuit complexity. In Proceedings of the 24th Conference on Foundations of Software Technology and Theoretical Computer Science, pages 336-347. Springer-Verlag, 2004.

[15] J. M. Hitchcock, A. Pavan, and N. V. Vinodchandran. Partial bi-immunity, scaled dimension, and NP-completeness. Theory of Computing Systems. To appear.

[16] J. M. Hitchcock and N. V. Vinodchandran. Dimension, entropy rates, and compression. Journal of Computer and System Sciences, 72(4):760-782, 2006.

[17] J.M. Hitchcock and E. Mayordomo. Base invariance of feasible dimension. Manuscript, 2003.

[18] D. W. Juedes and J. H. Lutz. The complexity and distribution of hard problems. SIAM Journal on Computing, 24(2):279-295, 1995.

[19] D. W. Juedes and J. H. Lutz. Completeness and weak completeness under polynomial-size circuits. Information and Computation, 125(1):13-31, 1996.

[20] L. A. Levin. On the notion of a random sequence. Soviet Mathematics Doklady, 14:1413-1416, 1973.

[21] L. A. Levin. Laws of information conservation (nongrowth) and aspects of the foundation of probability theory. Problems of Information Transmission, 10:206-210, 1974.

[22] M. Li and P. M. B. Vitányi. An Introduction to Kolmogorov Complexity and its Applications. Springer-Verlag, Berlin, 1997. Second Edition.

[23] W. Lindner. On the polynomial time bounded measure of one-truth-table degrees and pselectivity, 1993. Diplomarbeit, Technische Universität Berlin.

[24] M. López-Valdés and E. Mayordomo. Dimension is compression. In Proceedings of the 30th International Symposium on Mathematical Foundations of Computer Science, volume 3618 of Lecture Notes in Computer Science, pages 676-685. Springer-Verlag, 2005.

[25] J. H. Lutz. Dimension in complexity classes. SIAM Journal on Computing, 32:1236-1259, 2003. 
[26] J. H. Lutz. The dimensions of individual strings and sequences. Information and Computation, 187:49-79, 2003.

[27] E. Mayordomo. A Kolmogorov complexity characterization of constructive Hausdorff dimension. Information Processing Letters, 84(1):1-3, 2002.

[28] B. Ya. Ryabko. Coding of combinatorial sources and hausdorff dimension. Soviets Mathematics Doklady, 30:219-222, 1984.

[29] B. Ya. Ryabko. Noiseless coding of combinatorial sources. Problems of Information Transmission, 22:170-179, 1986.

[30] L. Staiger. Kolmogorov complexity and Hausdorff dimension. Information and Computation, 102:159-194, 1993.

[31] L. Staiger. A tight upper bound on Kolmogorov complexity and uniformly optimal prediction. Theory of Computing Systems, 31:215-229, 1998.

[32] A. K. Zvonkin and L. A. Levin. The complexity of finite objects and the development of the concepts of information and randomness by means of the theory of algorithms. Russian Mathematical Surveys, 25:83-124, 1970. 